\title{
37. Integrated Process Management System and RFID Directory Services
}

\author{
Masayuki Takata ${ }^{1}$, Eiji Arai $^{2}$ and Junichi Yagi ${ }^{3}$ \\ 1 The University of Electro-Communications Email: takata@cc.uec.ac.jp \\ 2 Osaka University Email: arai@mapse.eng.osaka-u.ac.jp \\ 3 Shimizu Corporation Email: junichi.yagi@shimz.co.jp
}

\begin{abstract}
This paper describes an implementation of the Integrated Process Management System, which includes manufacturing process management for building parts, and also construction process management at construction site. To observe the flow of the building parts, RFIDs are stuck to all parts to be managed, and several checkpoints, which we named "gates", are introduced within the coherent process through part-manufacturing and building construction. The requirements of the RFID directory services are also discussed.
\end{abstract}

\section{INTRODUCTION}

This paper describes an implementation of the integrated construction process management system, which includes both manufacturing process management features for building materials and also construction process management features at construction site, and a proposal of an RFID directory service agent.

Recently, RFIDs are getting popular in logistics industries and manufacturing industries. The process management system for building construction and building materials manufacturing must cover these two aspects, and the use of RFIDs in construction industries will make the trace-ablity of the building materials more accurate.

When the implementations described here are realized, in a case of some troubles found in building materials, other building materials are re-allocated for the order, and if requested in advance, other new orders are submitted to the materials manufacturers. Those systems enable efficient project management, by means of providing all information of the both material manufacturing and building construction processes to all of material designing, material manufacturing, building designing, and building construction sites.

Through this implementation project, we found that the presense of an RFID directory, which translates RFID identifiers into WIP identifiers and vise versa, is the very point within the manufacturing line control, because it should change its translation tables as WIPs are assembled and disassembled within a manufacturing line. 


\section{APPROACH}

\subsection{Paragraphs}

In this implementation, we aimed to confirm that the system operates properly on the whole. In order to make its information processing simple, the process management engine uses only typical durations to process each step in the manufacturing materials or installing them, and the bills of materials.

In order to trace WIPs(Work In Processes), we installed several checkpoints, which we named "gates", within the process through material-manufacturing and building-construction. On WIPs passing these gates, RFIDs are read and progress reports are collected to the process management system.

As the due time for passing the final inspection process of the installation to the building is deduced from the overall schedule of the building construction, the due time for passing each gates can be calculated from the given final due time and the typical durations from one gate to the next. In the other hand, when WIPs pass each gates, the estimated time for passing following gates can be calculated from the actual achievement time and the typical durations.

In these way, for each building materials types, we can obtain both due time for all demands passing all gates, and actual or estimated time for all WIPs. By associating each demands and each WIPs in the order of time passing a predefined gate for each building materials type, we carry out the allocation of demands and WIPs.

In the case of due time of allocated demands is earlier than estimated time of associated WIP passing by, we assume that tardiness is expected and some action is required, at the moment.

The due time for demands are re-calculated every time when the due for final inspection changes, and the estimated time of WIP passing by are recalculated every time when the WIP passes new gate. The reallocation of the demands and WIPs takes place, when either the list of demands or the list of WIPs sorted in order of time change.

\section{THE IMPLEMENTATION}

\subsection{Gates}

In order to trace WIPs, we have set up nine gates within both materialmanufacturing and building-construction processes, as follows.

- Design approved,

- Ordering raw material,

- Start processing,

- Assembling,

- Shipping out from the manufacturing plant,

- Carrying into the construction site,

- Distributing within the construction site,

- Installing building material in the building,

- Final inspection.

It is easy for the system to change the total number of gates, to change typical durations from gate to the next. It is also possible to differ the typical durations for 
calculating due time with the due for the final inspection gate from the durations for calculating estimated time to pass gates with action achievements information.

When the WIPs pass gates, following processes are took place.

- Reading RFID on the WIP,

- Converting to the WIP identifier,

- Logging the time passing the gate,

- Logging the physical position of WIP,

- Logging the result of the post-process testing (optional).

These data are accumulated within an actual achievement database resides in the shared data space, described later.

\subsection{Tracking Works with RFIDs}

At each gate, the system gathers actual achievement information by means of RFIDs. In this implementation, we assumed to use read-only type RFIDs with 128 bit length identifiers.

Generally speaking, as each building materials consists of multiple parts which are manufactured independently in the manufacturing line, single building material may contain multiple RFIDs in it. On reading RFIDs of a building material, some of multiple RFIDs may respond and some may not, but the tracking engine should handle these information properly in any case. In some cases, an assembled WIP may be dis-assembled to find much more matching combination.

So, the tracking engine should have following features.

- Identifying the WIP from partial RFIDs information.

- Keeping RFID identifiers of all parts consisting the WIP.

- Keeping tree-structured information including assembling order and part structure, for the case of dis-assembling and re-assembling.

Furthermore, it is expected that reading some particular RFIDs instead of entering some information manually, such as operator's name, physical location of the workcell, and others. So, the tracking system can judge whether given identifier represents some WIP or not.

\subsection{Allocation}

In this implementation, we use simple algorithm described in the Section 2 to allocate demand to corresponding WIP.

The basic data, which are the typical durations from one gate to the next or previous gate and the bills of materials, are given by initializing agent and stored in the shared data space among multiple processing agents, described later. In this implementation, the typical durations for due date deduction may differ from the typical durations for calculating estimated times passing following gates. Both durations are defined for all demand and WIP types as default values, but the users can define other values to override them for respective types.

We named the lists of same type WIPs, which are arranged in the order of actual expected time passing a predefined gate, as "preceding list." In other hand, we named the list of same type demand, which are arranged in the order of due time at a predefined gate, as "priority list." Their priority is defined only by the due time order, and we ignore any other value-related information which may affect on priority of demands. 
All demands and WIPs are processed in building material type by type, and in the case of the material type made from multiple parts, the demands of those parts is newly created according to the bills of materials at the gate at which those parts are assembled together. The due time of the parts is set as same as the due time of the combined WIP at the same gate. The estimated time of one WIP to pass the assembling gate is calculated as the latest one of the estimated time of its parts to pass the gate.

In order of simplify the allocation algorithms, we assumed that there is no part types which are assembled into multiple building materials, in order to omit the idea of priority.

\subsection{User Interface}

In this subsection, we describe the user interface screens implemented. The user interface of this system was developed as the application of World Wide Web system, in order to make them accessible from not only desk-top computer systems but also portable data terminals.

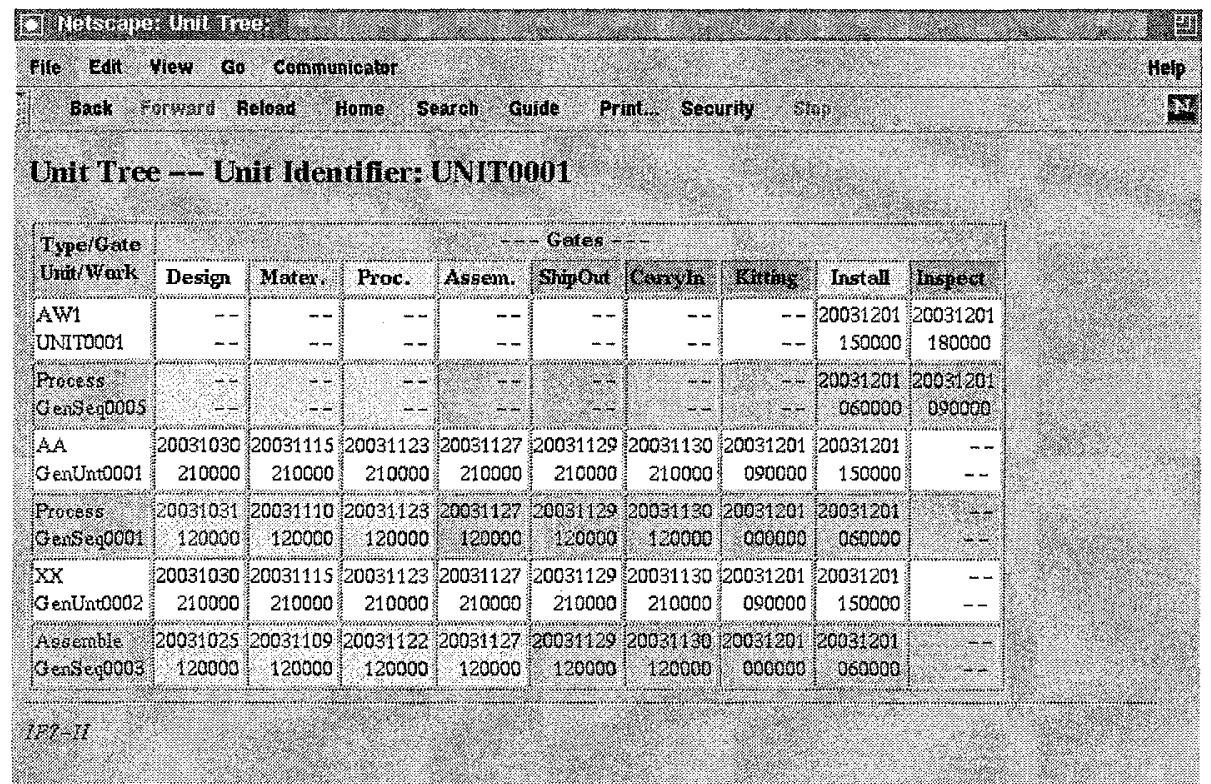

Figure 1: Due time to passing gates and estimated time passing following gates

Figure 1 shows the status display for one particular association of the demand and the WIP, including the parts which demands and WIPs consists of. In each table entry, the upper row contains date information in the format of YYYYMMDD, and the lower one contains time in the format of HHMMSS.

The table entries with while background color show the due time of the demand for that gate, those with green background color show the actual achievement time of the WIP to pass the gate, and those with blue background color show the estimated time of the WIP to pass the gate calculated from the actual achievement time for the last gate passed and typical duration time.

In this table, the demands and the WIPs are shown in the preceding list or the priority list order. 
The neighboring entries consisting upper demand line and lower WIP line show the allocation of demand and WIP. This allocation is subject to change, and the indications changes when due dates are changed or WIP passes new gate. In the case of tardiness expected in some allocated pairs, their actual achievement date and time are shown in red characters.

Figure 2 shows the status display for one particular type of building materials, including the parts which demands and WIPs consists of.

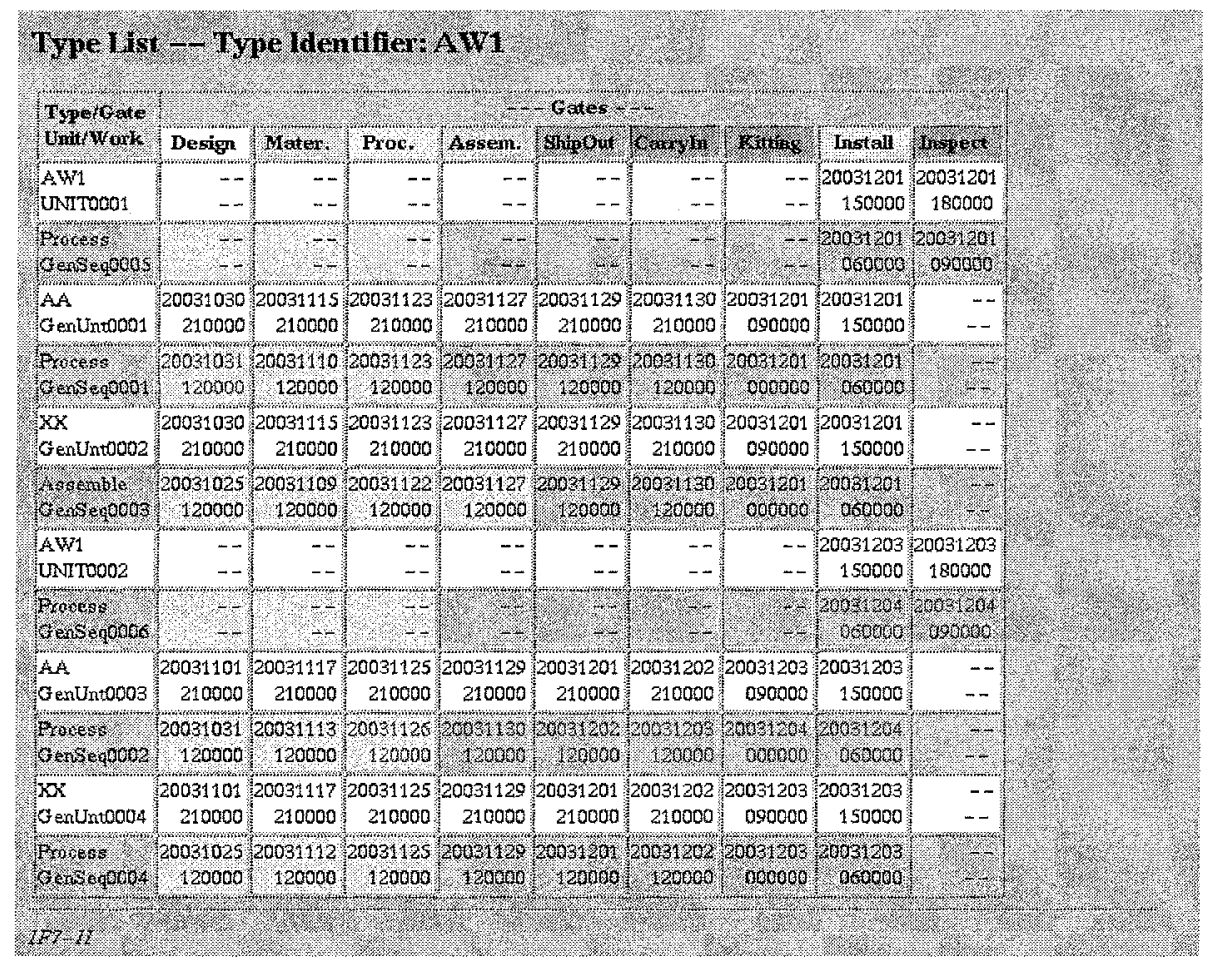

Figure 2: Status display for all works of specified building material type

This example shows that two building materials are currently under processing, and one WIP recovered its tardiness at the first gate, by shorten the duration for processing from the first gate to the second, but another WIP has delayed at the last gate it passed and tardiness at the gate for the final inspection is expected.

In order to recover such situations, one of following behavior is feasible.

- To shorten the duration from the gate to the next, as the first part has been done.

- To postpone the due time for the final inspection of the building materials installation.

Figure 3 shows the case of postponing the due time of the final inspection of the unit with unit-ID UNIT0001 for four days. As the result, the due time of the unit UNIT0001 and that of the unit UNIT0002 are reversed, and the parts set which are going to be used for those units are exchanged. 


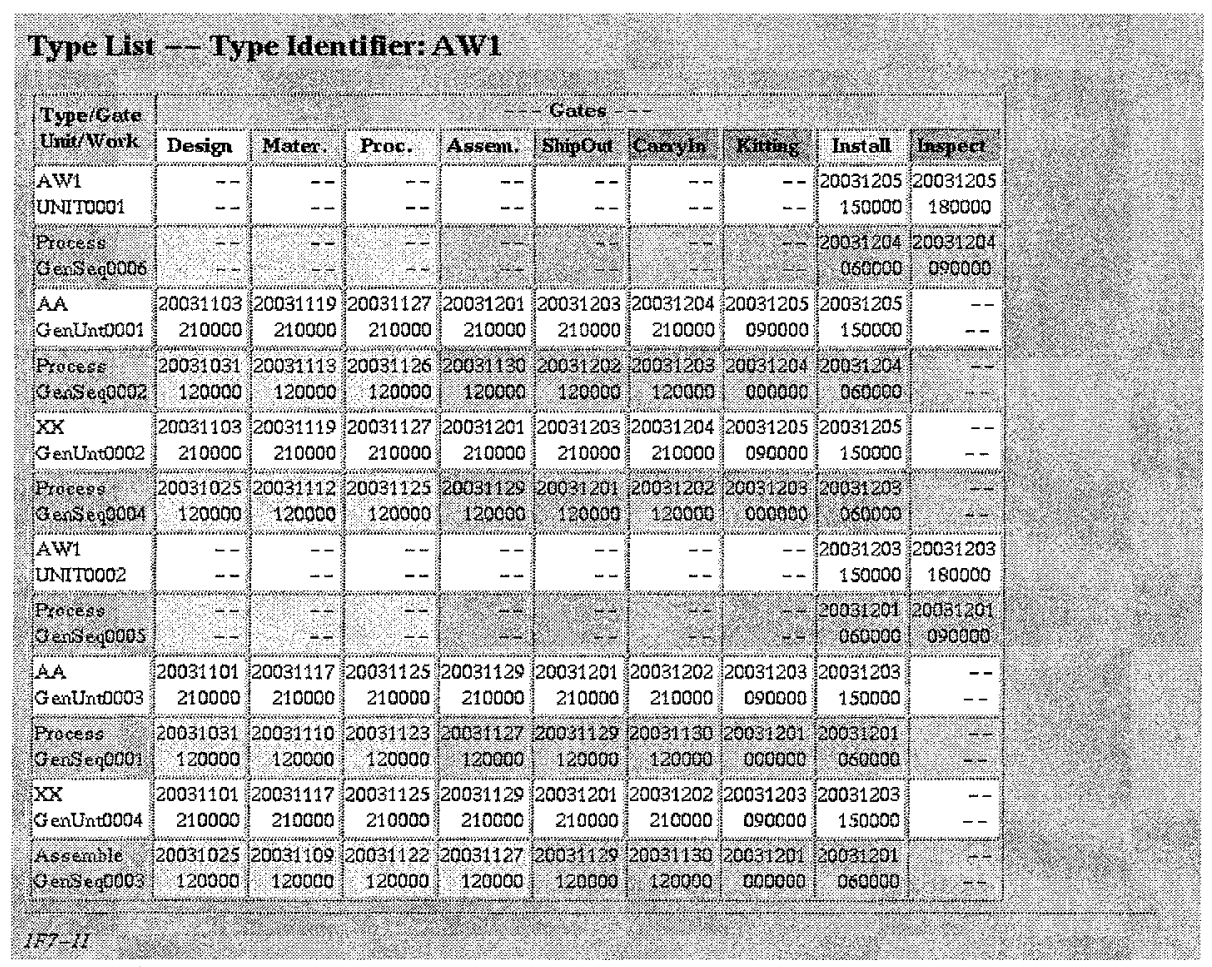

Figure 3: Status after changing due time

\section{RFID DIRECTORY SERVICES}

As we mentioned in the preceding section, in the manufacturing line control systems using RFIDs, we have to translate RFID identifiers into WIP identifiers and vice versa. This information consists of both associations of WIP identifier and RFID identifiers and tree-structured information including part structures.

As the parts are assembled and rarely but also disassembled in their manufacturing process, the set of RFIDs each WIPs are containing varies as their process progress, and the translation table should be updated as WIPs are processed. Basically, the translation table contains set of associations of a WIP identifier and a set of RFID identifiers. But once some parts with RFIDs are assembled and joined together, the biggest WIP containing the RFID should be answered to a request translating RFID identifier into WIP identifier.

As we can not change association information preparing cases of disassembly, the inclusion relations among WIP identifiers are maintained separately from the associations of WIP and RFID identifiers. The structure of this relation is similar to the structure of the bill of materials, and it is rather easy to trace the changes of an WIP through assembly process. But in the cases of the disassembling WIPs to change some of their components to re-assemble and to pass the inspection, it is very difficult to enumerate all the way to disassembling each WIP.

\subsection{Basic Functionalities}

The RFID directory service should have following functionalities: 
- Keeping set of RFID identifiers, which are NOT used in order to identify WIPs. For example, RFIDs to identify operators, physical locations, gates in the manufacturing process, and others.

- Keeping associations between a WIP identifier and a set of RFID identifiers.

- Keeping the information of including relations among WIP structure, which can be obtained from the bill of materials.

- Tracking each WIP's current structure and its consisting parts.

\subsection{Very Requied Functionalities}

As this RFID system is used within manufacturing processes, the objects identified by RFIDs are assembled and disassembled. So the directory service should have following functionalities in order to handle joining and separating parts.

- Operation on joining some WIPs, which includes modification of WIP's structure information or creating a new WIP identifier and initialize its structure information.

- Operation on disassembling an WIP, which includes not only the modification of WIP's structure, but also includes finding the description of separation by scanning RFID identifiers. In many cases, as the disassemble process is not regular one in order to recover some difficulties introduced within preceding assemble processes, the set of the parts to be removed is not predictable and may varies in cases. This is because the RFID directory service should have abilities to guess members of some resulting sets themselves and their structures.

In the area of the life-cycle engineering, the reusing of the used parts will play a vital role in the parts management. In such application, the management of the parts should be based on technology of RFIDs, or something alike which can identify each parts from other same type parts, in order to trace back parts history and the past operation conditions, diagnostics results and maintenance logs.

For these purpose, further research is required on the RFID directory service agent, especially in the area of handling disassembling processes and old history management.

\section{THE INFRASTRUCTURE SYSTEM}

In this study, we used the system named "Glue Logic" (Takata and Arai, 1997) (Takata and Arai, 2001) (Takata and Arai, 2004) as the infrastructure to support multiple-agent processing system, in order to implement a kernel of MES (Manufacturing Execution System) controlling manufacturing processes.

The Glue Logic, which is designed and developed by Takata Laboratory of the University of Electro-Communications in Japan, includes an implementation of the active database and the network transparent programming environment, and supports data processing in the event driven programming paradigm.

Figure 4 shows the overall structure of this implementation.

\subsection{Active Database}

The active database is a subclass of the database systems, of which databases have an abilities to behave when it finds some changes of its contents, without waiting for external actions. 


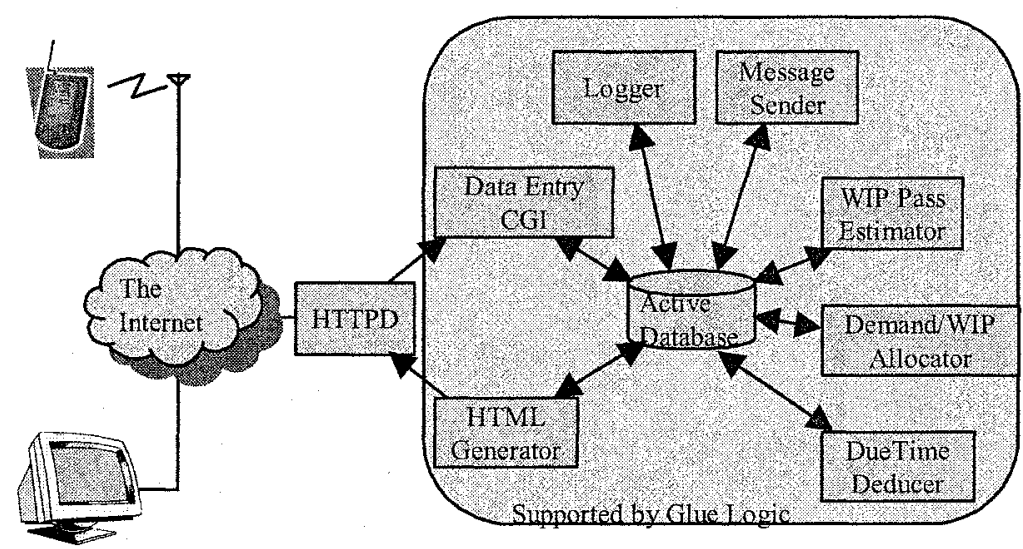

Figure 4: Overall Structure of the Implementation

The change of the contents includes

- when data is changed,

- when some relations are formed,

- when some new data become available.

and the behavior executed on these incidents includes

- changing contents of the database,

- calculating certain expression and assign the result into some variable,

- sending message to some client agents.

\subsection{Aims and Functionalities of the Glue Logic}

The Glue Logic is designed to make building manufacturing work-cell control systems easy and flexible, and also coordinates agents by means of followings;

- Providing field of coordination

- Implementing shared data space among agents

- Virtualizing agents within the name space of shared data

- Controlling message passing among agents

- Implementing mutual execution primitives

- Prompting agents to start processing

- Adapting control systems to real-time and network processing environment

As the Glue Logic supports event notification and condition monitoring features based on active database scheme, users can easily build real-time and event-driven application agents, only waiting for notification messages from the Glue Logic.

Each agents in an application system can be developed concurrently, and can be added, deleted or changed freely without modifying other existing agents. As the result of these, the Glue Logic compliant agents are easy to re-use, and the users can build large libraries of application agents.

\subsection{Role of the Glue Logic}

In this implementation, the flow of its data processing is as follows.

- When a WIP reaches new gate, or when a due time of a demand changes, the corresponding agent is activated via a Common Gateway Interface (CGI) for the 
World Wide Web user interface. These agents updates the actual achievement records for WIPs or the due time requirement records for demands. These records are kept in the shared data space in the Glue Logic.

- When data within the shared data space is changed, some messages are sent from Glue Logic to agents which have already subscribed to the data items. This time, the agents keeping preceding lists or priority lists are informed, and update those contents.

- When the contents of preceding lists or priority lists are changed, the notification message is sent from Glue Logic to the allocation agent. The allocation agent reads preceding lists and priority lists, and then makeup associations between demands and WIPs.

As described above, the Glue Logic plays role of the conductor or alike of the whole system.

\section{EVALUATION}

\subsection{Evaluation on the performance}

We implement the system on Sun Netra T1 processor running on Solaris 8 OS. Through this implementation, we found following performance considerations.

- Re-allocation of the demand hardly took place unless due time of final inspection changed or some WIPs pass other preceding WIPSs.

- In many cases, as there are less than tens of WIPs concurrently being processed in both material-manufacturing and building-construction sites, re-allocation of the demand takes only a few seconds. But in the case of building materials with complex BoM (bill of material), as the re-allocation process occurs in multiple stages, it may takes more than ten seconds.

- It may takes a few minutes for operators to input data for gate passing process or changing due time. So, some features to inhibit processing during data entry may be required to prevent needless data processing.

\subsection{Evaluation on the extend-ability}

We used multiple agent support system in the implementation, in order to ease future extension of functionality. From this point of view, we found followings.

- As all information on the actual process achievements are kept within a database, any other agents can utilize these data for processing and user interface purposes, as follows:

- displaying status of building materials within building floor plan chart

- sending e-mail to notify shortage or tardiness of some materials, within a few seconds.

- It seems to be appropriate that the conversion from the identifier of RFID to building material identifier should be done by specialized subsystem in the management system. There may be many RFID classes representing objects other than WIPs.

\subsection{Evaluation on the limitation}

In this implementation, we introduced some limitations to simplify the system. They are as follows. 
- There is no problem solving engine to minimize cost. In order to find best solution of re-allocation, it is required to minimize cost to re-distribute building materials, or it is required to determine which WIP to be scraped. Solving this problem may need massive computational power, because there may be combinational explosion. In the other hand, we can not define the function to evaluate the cost itself clearly in the practical world. For example, in many cases, two types of building materials are not equivalent in their priority.

- There is no clear decision rules to be embedded within the system. Some incidents can be processed automatically without human interventions, but some require human approvals. There is no clear border and the best way depends on its environment.

- In the calculation of the due time and estimated time to pass, we use "typical durations." These values are constants and have less accuracy. In the following research, we have to link with APS (Advanced Planning and Scheduling) systems and MRP (Manufacturing Resource Planning) systems.

\section{CONCLUSION}

Through this implementation described above, we found that the integrated process management system including both part-manufacturing and building construction is feasible enough.

Especially, related to the use of RFIDs, we recognized the importance of the management agent, which we called RFID directory service agent in this paper. This agent keeps not only set of association between WIP itselves and the RFIDs included in the WIP, but also the inclusion relations among WIPs.

In coming years, we would like to test next implementation at the actual manufacturing and construction sites.

\section{Acknowledgments}

This research activity has been carried out as a part of the Intelligent Manufacturing Systems (IMS) international research program: "Innovative and Intelligent Partsoriented Construction (IF7-II)". We appreciate the kind guidance of each members of this project.

\section{REFERENCES}

Takata,M., Arai,E. (1997) The Ghue Logic: An Information Infrastructure System for Distributed Manufacturing Control. Proc. of the Int'l Conf. on Manufacturing Milestones toward the 21st Century, pp.549 - 554, Tokyo, Japan, July 23-25, 1997.

Takata,M., Arai,E. (2001) Implementation of a Layer Structured Control System on the "Glue Logic". In Mo, J., Nemes, L. (eds.) Global Engineering, Manufacturing and Enterprise Networks, Kluwer Academic Publishers, pp 488-496

Takata, M., Arai,E. (2004) Implementation of a Data Gathering System with Scalable Intelligent Control Architecture. Arai, E., J. Goossenaerts, F. Kimura and K. Shirase (eds.) (2005) Knowledge and Skill Chains in Engineering and Manufacturing: Information Infrastructure in the Era of Global Communications, Springer, pp 261-268 